\title{
Increasing the sustainability of coatings for precoated metal
}

\author{
C. Lowe \& J. T. Maxted \\ Becker Industrial Coatings Ltd, UK
}

\begin{abstract}
Sustainable coatings for pre-coated metal can come in many guises, for instance the raw materials themselves can be derived from sustainable sources such as biomass. In addition they can be smart, which means having some degree of functionality; for instance, they can help improve the thermal comfort within a building by reflecting or absorbing solar radiation depending upon global location and whether the interior needs to remain cool or achieve a particular level of warmth. Furthermore, the aesthetics of a building can be improved by resisting dirt. All of these smart effects combined with more sustainable sourcing can be used to obtain BREEAM or LEED points.

Keywords: bio-sourced coatings, solar reflection, solar absorption, dirt pick up resistance.
\end{abstract}

\section{Introduction}

Pre-coated metal is used extensively by the construction industry; it is especially prevalent in Western Europe on warehouses, retail outlets and factories. In Eastern Europe and Scandinavia there is extensive use made of the technology for domestic roofing. The main substrates used in these areas are hot dip galvanised steel and aluminium. The latter tends to be used more on prestigious projects. There are many claims made by the steel and aluminium producers about the sustainability of the metals because they are relatively easily recycled with lower energy requirements than if they are made from their ores. However, there is a question mark over the sustainability of the paints used.

The paints for pre-coated metal have developed over the past 40 years from alkyds (which are partially derived from plants) and PVC plastisols to the more modern super-durable polyester/melamine systems. These more modern coatings 
are based on polymers almost exclusively derived from petroleum. How then can they be made more sustainable?

Firstly the bio chemical industry is rapidly developing methods to produce the basic building blocks of polyesters from plants, namely glycols and di-acids. Some are already familiar to the synthetic resin chemist and subsequently the paint formulator. Others are less familiar and so the resulting polyesters have different properties which require evaluation.

Secondly, coatings can be encouraged to have a degree of functionality; that is they react to environments or have properties that help them act in a certain way in specific environments.

\section{Sustainability}

What is sustainability? A dictionary definition of sustain is "to endure". The same dictionary defines sustainable as that which conserves an ecological balance by avoiding depletion of natural resources. However there are more definitions. For instance;

"Sustainable development is development that meets the needs of the present without compromising the ability of future generations to meet their own needs" [1].

"To be sustainable a company must first make a Profit, it must look after its People and it must look after the Planet" [2].

Both definitions have similar themes but they vary in the focus of their emphasis. It is clear that the second definition has been devised by a commercial organisation because if a company is going to change the world on an on-going basis then it must not go out of business!

So what are the strategies that could be followed by the coatings industry to ensure a more sustainable future? A paint company can start by looking inwardly and make a determined effort to reduce energy usage both in the manufacture and the transportation of its products. Similarly waste, VOCs and carbon dioxide production can all be reduced to minimum levels [3]. It should look concurrently at its products and how they can be sourced more sustainably: that is the reliance on petroleum derivatives should be reduced by focusing on materials derived from or with the help of plants (preferably waste from other processes). Some pigments are already entirely derived from waste materials. Titanium dioxide is the most extensively used pigment and although it is not made exclusively from waste materials titanium dioxide manufactures are working to make their processes more sustainable. Furthermore coatings can deliver a sophisticated range of functionalities which help to reduce the energy required to cool or heat buildings and maintain a clean surface [4].

Pre-coated metal is a product that is used extensively in the construction industry because it has a consistent factory-produced nature, its use ensures that building envelops can be erected rapidly, it is durable but lightweight and, perhaps best of all, it is cost efficient. The base substrate can be easily recycled, be it steel or aluminium, but are the steps mentioned above being taken to ensure that the paints themselves are more sustainable? 
This paper will consider sustainable raw materials that are currently available and through the concept of glass transition temperature (Tg) it will explain why there are limitations. It will go on to discuss functionality and how one example uses $\mathrm{Tg}$ and other surface properties to deliver self-cleaning surfaces.

\section{The concept of glass transition temperature}

One of the first natural polymers to be used was rubber. Sulphur was added to natural rubber during vulcanisation in order to form chemical bridges between the long molecules that constitute the material. This crosslinking toughens the rubber for use in tyres etc. If rubber is dipped into liquid nitrogen it becomes hard and brittle like a glass. The mid-point of the temperature range over which this change occurs is therefore known as the glass transition temperature. All polymers have a glass transition temperature ( $\mathrm{Tg})$; it is a key property which determines how the polymer behaves at any point on the temperature scale. For instance polystyrene has a Tg of around $105^{\circ} \mathrm{C}$ which means below this temperature it is hard and brittle making it suitable for use in disposable cups that can withstand boiling water without losing shape or functionality. Polymers for coatings also have Tgs which determine the performance of the coatings over the temperature ranges they operate in. Automotive systems generally have Tgs well above room temperature to ensure they are not too soft and easily damaged. However the result is that they are relatively brittle and prone to impact damage.

Polymers will change from being glassy to rubbery over a temperature range, because they are made up of molecules of different sizes which require different amounts of energy to move: the smaller molecules adopting a more mobile state at temperatures below those that would encourage larger molecules to move. However it is convenient to use one temperature value to define this range and many workers in the field use the midpoint of the range to quantify this property, although the onset temperature is also quite common. A common method used to study Tg is Differential Scanning Calorimetry. This technique detects changes in heat capacity; a typical thermo gram is shown in Figure 1.

The predominant polymers used for coil coatings are polyesters, cross-linked with either melamine resins or poly-isocyanates. The properties of these paints are

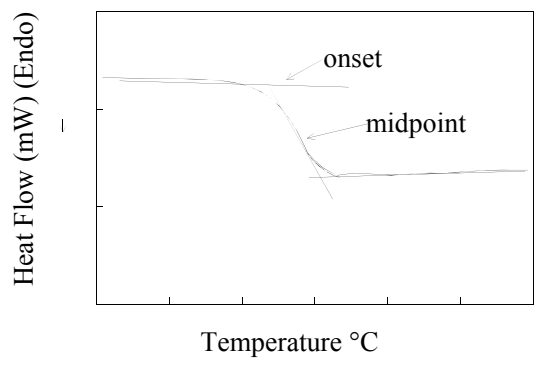

Figure 1: Typical thermo gram from a DSC. 
determined by their crosslink densities, their Tgs and the temperature at which stress is applied. Paints for pre-coated metal are the result of careful optimisation because they need to be formable so that they can undergo the roll-forming process, abrasion resistant so that they do not mark during the same operation, hard so that they are scratch resistant and durable.

\section{The availability of bio-sourced raw materials}

\subsection{Monomers available for polyesters from biomass}

There are a range of petroleum based glycols (diols) and di-acids that can be reacted together to make the relatively low molecular weight polyesters used for pre-coated metal. One or more of the three aromatic acids are commonly found in polyesters used in these systems, the determining factors being cost and durability. Ortho phthalic acid is a cost effective aromatic di-acid, whereas isophthalic acid is more expensive but improves the durability of the coating.

Glycerol, which is formed when fatty acids are released from fats and oils made by animals and plants, can be transformed into 1,3 propan-diol [5] which can be used in the preparation of polyester resins. Succinic acid is an aliphatic di-acid which e-coli and other bugs metabolise. There is a pilot plant in Northern France run by Bio Amber [6] which has been set up to produce 2000Tpa of succinic acid by a fermentation process that uses sugars as the raw material. Their aim is to produce bio-based succinic acid at no greater cost than that currently achieved with the petroleum based equivalent. 1,4 Butane diol can be made from succinic acid and is thus another raw material that can be bio-sourced.

Until recently the synthesis of phthalic acids from bio-sourced raw materials was a long way off on the horizon; however the cola companies have been pushing hard for a bio-sourced terephthalate to complement the bio sourced ethylene glycol that has been used in their partially bio-sourced PET for the past few years. Several companies have proposed routes to bio sourced phthalic acids but currently nothing is available. Hence polyester resins are rarely $100 \%$ bio-sourced, still relying on the presence of petroleum based aromatic acids to achieve the required $\mathrm{Tg}$ but the future is bright even for these monomers.

\subsection{Feed stocks now and in the future}

Although sugar has been the most commonly used bio-feed stock there is a realisation that bio-sourced polymers should, as much as possible, be derived from waste products from other processes. Straw and saw dust are two examples that immediately spring to mind. The main components of wood are cellulose and lignin. In fact cellulose is the most common bio polymer followed by hemi cellulose and then lignin. Cellulose itself is a polysaccharide, which is it is made up of many sugar molecules. Many companies are now trying to use cellulose as a bio-feed stock for the range of chemicals mentioned in place of sugar. 


\subsection{Costs}

Although comparative prices with petroleum based equivalents are the stated aims of many of the companies involved in the production of bio-sourced raw materials, it will be apparent that these materials are being synthesised using new routes. They will not be available immediately in volumes associated with the commodity chemicals currently used and will therefore be more expensive. Thus despite of the desires of the suppliers of bio-based chemicals, sustainability will cost. But at least it won't cost the earth!

\section{What bio-sourced coatings are currently available?}

Beckers is in the process of introducing a range of coatings based on bio-sourced polyesters which eventually should cover the entire spectrum of mechanical and durable properties. Understandably we have started with systems that comply with the least stringent properties with the intention of gradually increasing the range to include more durable or more formable coatings.

Last year a collection of internal quality products was introduced including a liner, a primer and a backer. The systems are melamine cross-linked polyesters based on a resin with a $100 \%$ bio-content. The mechanical performances of the cured coatings applied to chromate free pre-treated HDG are shown in Table 1. A photograph of the backing coats' $\mathrm{T}$ bend performance is shown in Figure 2.

Table 1: Mechanical performance of bio-based backer compared to standard.

\begin{tabular}{|l|c|c|c|}
\hline Test & Standard & Std backer & Bio backer \\
\hline Total dry film thickness & $E N 13523-1$ & 12 microns & 8 microns \\
\hline Gloss $\left(60^{\circ}\right.$ head) & $E N 13523-2$ & $34 \%$ & $41 \%$ \\
\hline Pencil hardness & $E N 13523-4$ & H min & F-H min \\
\hline Reverse impact 80"lbs & $E N 13523-5$ & $\begin{array}{c}\text { No crack } \\
\text { /No removal }\end{array}$ & $\begin{array}{c}\text { No crack } \\
\text { No removal }\end{array}$ \\
\hline Cupping test (7 mm draw) & $E N 13523-6$ & $\begin{array}{c}\text { No crack } \\
\text { /No removal }\end{array}$ & $\begin{array}{c}\text { No crack } \\
\text { /No removal }\end{array}$ \\
\hline T-bend (no cracking) & $E N 13523-7$ & 2.5T No cracking & 2.5T No cracking \\
\hline T-bend (no removal) & $E N 10169-$ & 1.5T No removal & 2.0T No removal \\
\hline Solvent resistance & EN13523-11 & 100+ MEK rubs & $100+$ MEK rubs \\
\hline
\end{tabular}

Early this year an exterior quality topcoat and suitable primer were offered to the market. The resin used was not completely bio-based because aromatic acids were necessary to achieve hardness and outdoor durability performance. The result was a topcoat that has a bio-content of more than $25 \%$ based on the dried film. Based on gloss and colour retention changes on weathering for a range of colours exposed for 2000 hours in UV-A cabinets, it is clear that the coatings can be classified as $\mathrm{R}_{\mathrm{Uv}} 2$ quality ( $>30 \%$ gloss retention). Changes in gloss retention with exposure time are presented in Figure 3 for white and dark green paints. The mechanical properties of 5 colours are presented in Table 2. 


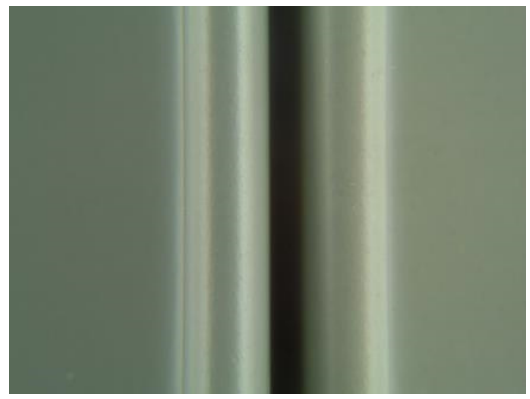

Figure 2: $\quad 2.5 \mathrm{~T}$ bend on backing coats bio based RHS.
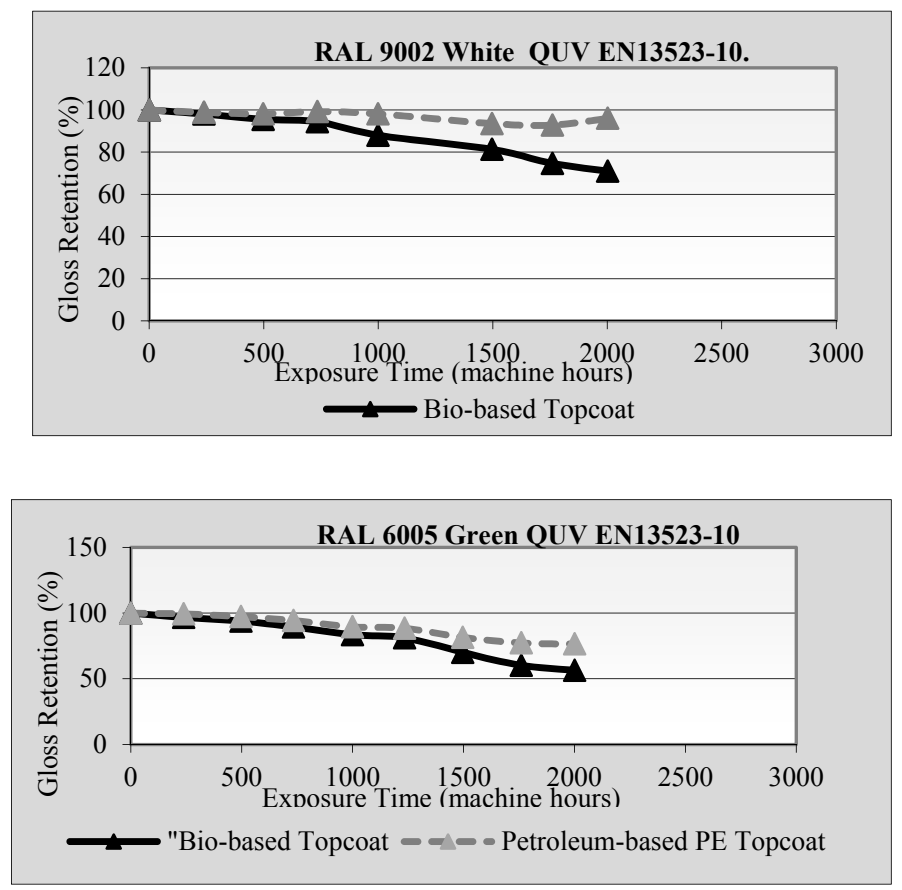

Figure 3: Gloss retention results of RUV2 class bio based polyester for 2 colours after 2000 hours accelerated weathering.

There is a growing demand for products that can be classified as more sustainable. Bio-derived coatings can be considered as such and Beckers is committed to increasing both the bio content and the overall paint performance over the coming years to ensure that they will be considered for point scoring under the BREEAM scheme. Caravan designers and Domestic Appliance OEMs are also asking for products that help them achieve greater levels of sustainability and we are as always willing to help. 
Table 2: Mechanical tests on standard and bio-based $\mathrm{R}_{\mathrm{UV}} 2$ PE topcoats. All coatings resisted over $100 \mathrm{MEK}$ double rubs and both $7.5 \mathrm{~mm}$ slow draw and $9 \mathrm{~J}$ reverse impact tests with no tape pick off.

\begin{tabular}{|l|c|c|c|c|c|}
\hline Category & Colour & $\begin{array}{c}\text { T-bend } \\
\text { adhesion }\end{array}$ & $\begin{array}{c}\text { T-bend } \\
\text { flexibility }\end{array}$ & $\begin{array}{c}\text { Pencil } \\
\text { hardness }\end{array}$ & Coin scratch \\
\hline & & \multicolumn{2}{|c|}{$($ EN 13524-7) } & EN 13524-4 & In house \\
\hline Standard & White & 1.5 & 1.5 & H & Std \\
\hline Bio & White & 0.5 & 1 & H & Softer \\
\hline Standard & Brown & 0.5 & 1.5 & H & Std \\
\hline Bio & Brown & 0 & 1 & H & Softer \\
\hline Standard & Red & 1.0 & 1.5 & H & Std \\
\hline Bio & Red & 0.5 & 1 & H & Softer \\
\hline Standard & Blue & 0.5 & 1.5 & H & Std \\
\hline Bio & Blue & 0 & 1 & H & Softer \\
\hline Standard & Dark green & 1.0 & 1.5 & H & Std \\
\hline Bio & Dark green & 0 & 1 & H & Softer \\
\hline Standard & Light green & 1.0 & 1.5 & H & Std \\
\hline Bio & Light green & 0 & 0.5 & H & Softer \\
\hline
\end{tabular}

\section{Functional coatings}

For as long as they have been available, paints and other surface coatings have had the dual function of protecting the underlying substrate as well as making it look aesthetically pleasing. Thus coatings for metals have been developed to inhibit corrosion whilst coatings for wood have prevented degradation by moisture/UV light. But surface coatings can be engineered to have other functions in addition to these more traditional features. As mentioned previously the coatings sold into the pre-coated metal market already demonstrate a range of properties almost taken for granted. They are; formable, relatively abrasion resistant, UV light resistant and stain resistant. It was shown recently [7] that polyester melamine coatings are toughest around their Tg but scratch resistance requires a high degree of hardness, hence these two properties will forever need to be optimised unless of course the roll former can form warm (Figure 4).

\subsection{Coatings that can control thermal comfort}

When carefully formulated, coatings can reflect the Near Infra-Red radiation arriving from the sun and thus help cars and buildings to stay cool. That is the coating formulator can make black paints behave as though they are white by reflecting this part of the sun's energy. The main instrument used for this is pigment choice. Cool roofing is now a common material of choice for architects in the US where it can help gain LEED points and provide compliance with US State and Federal schemes. This technology is also gaining ground in the Middle East as well as countries like Malaysia who also have Green Building programmes and building sustainability rating schemes in place This technology offers the 


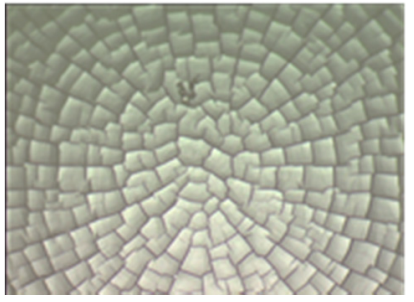

$\mathrm{T}=39^{\circ} \mathrm{C}$

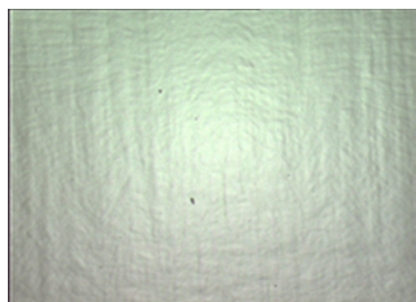

$\mathrm{T}=50^{\circ} \mathrm{C}$

Figure 4: Comparison of results from Erichsen slow draw test carried out on a polyester melamine topcoat with a $\mathrm{Tg}$ of $50^{\circ} \mathrm{C}$.

architect a wide pallet of colours for solar reflecting surfaces, they no longer have to be white. One parameter increasingly used to quantify the degree of envelope heat build-up is the Solar Reflectance Index or SRI. This number is comprised of the sum of amount of light absorbed at wavelengths from the near UV to the start of the mid infra-red, the emissivity and amount of cooling caused by the wind. Recently, Beckers LTD UK Laboratory became the first laboratory in Europe to gain ISO 17025 accreditation for its Radiation Property Testing Facility which measures SRI. Figure 5 shows how the NIR reflectance spectra of similar colours can vary depending upon pigment choice.

NIR reflectivity is a benefit in hot countries with high levels of sun light but with a little knowledge of material science the formulator can turn this phenomenon on its head and have a white that behaves like a black. The high solar absorbance of dark colours has been used to great effect on transpired solar collectors such as Solar Wall ${ }^{\circledR}$ (Figure 6) by Conserval. The CA group demonstrated that it is possible to heat a building just using solar radiation in early spring in the UK [8]. Figure 7 presents the NIR absorption spectrum of a modified

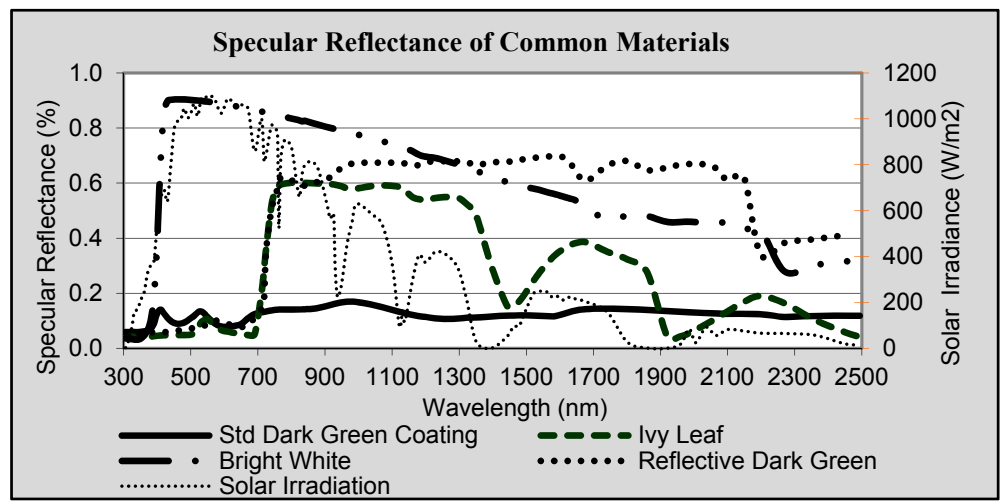

Figure 5: Reflectance spectra of a white paint, a green leaf and 2 green coatings. 
green colour which is in place on the Sustainable Building Envelope Centre in Shotton, North Wales.

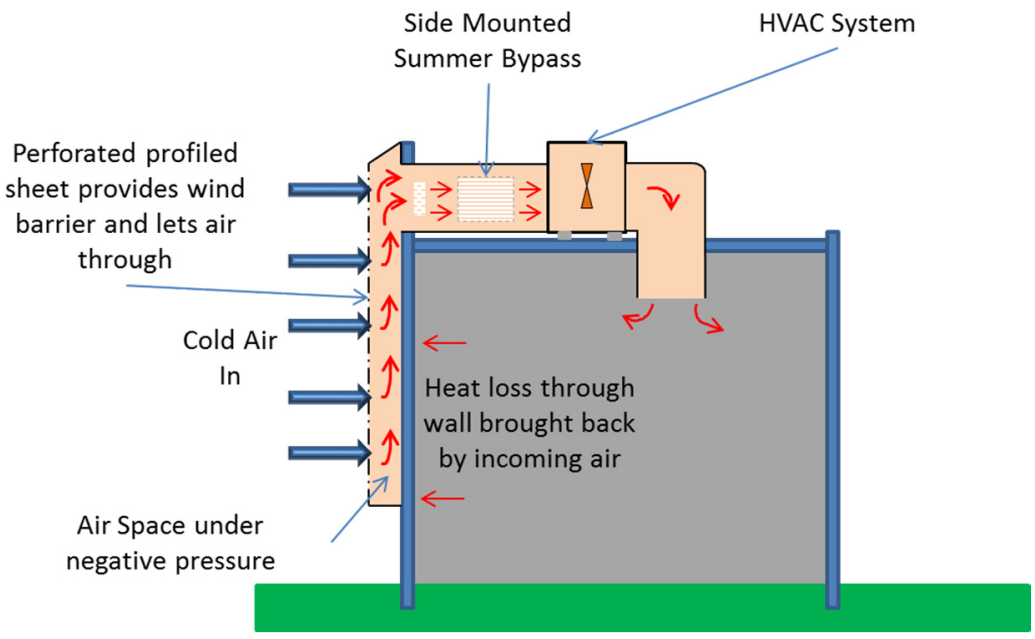

Figure 6: $\quad$ Solar Wall $^{\circledR}$ from Conserval.

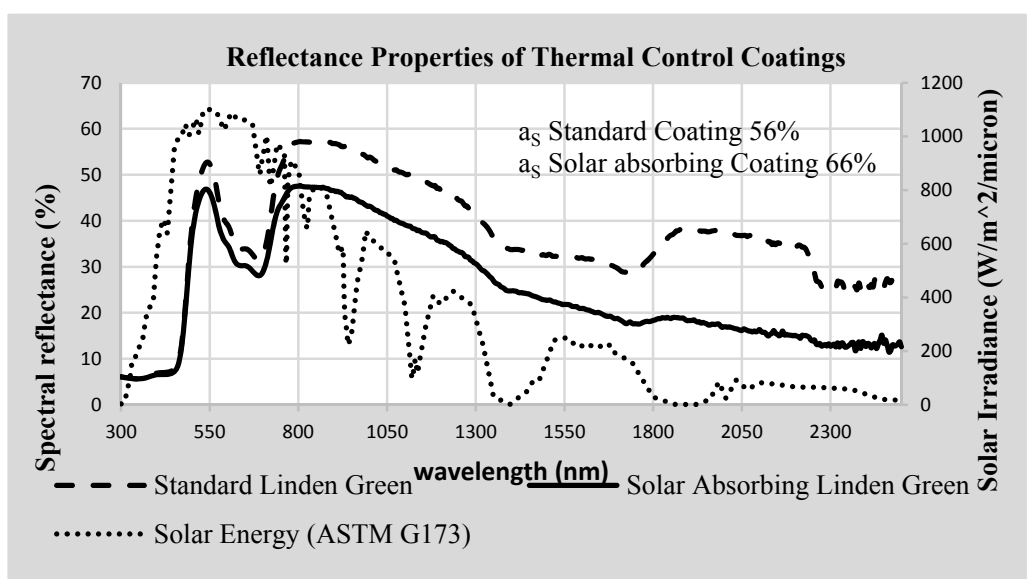

Figure 7: NIR spectra of a solar absorbing Linden Green and the standard.

\subsection{Self-cleaning coatings}

Another way to help the built environment would be to have surfaces that clean themselves. The Lotus flower stays clean by having a nanostructured hydrophobic surface [9]. This surface has so few contact points that any dirt particle cannot 
adhere very well, especially as the surfaces that it does touch are so slippery that no significant adhesion can be achieved.

However this is not the only route to a dirt free surface. Dirt particles of less than $1 \mu \mathrm{m}$ can sink into the paint, making it impossible to clean. This occurs if the $\mathrm{Tg}$ of the paint is lower than the service temperature and so the coating is above its Tg the majority of the time. To explain these results, it is necessary to consider how particles of $0.5-10 \mu \mathrm{m}$ diameter adhere to surfaces. Ramai et al [10] concluded that Van de Waals forces were the predominant cause of particle adhesion. However their work with materials above Tg suggested that adhesion induced engulfment would occur if the particles were small enough. Larger particles would remain on the surface. Thus they showed that this could happen for $4 \mu \mathrm{m}$ glass beads on plasticised polystyrene whereas $11 \mu \mathrm{m}$ beads remained proud of the surface (Figure 8).

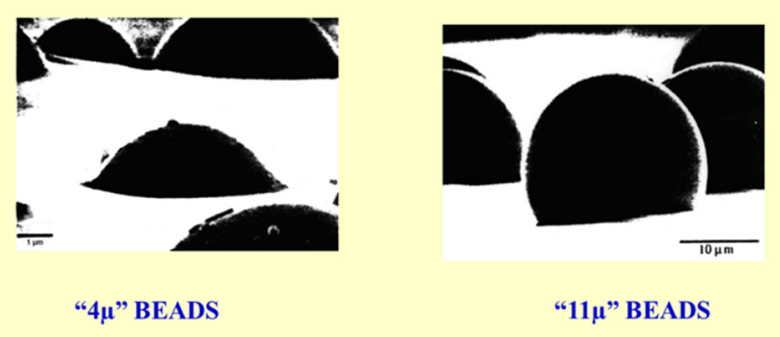

Inert glass beads on plasticised polystyrene

Figure 8: Glass beads of different sizes on plasticised polystyrene.

The elastic modulus of polyester coil coatings varies with temperature as does the modulus of all other polymeric systems. Dynamic Thermal Analysis has shown [11] that below Tg polyesters have a modulus around $10^{9.3} \mathrm{MPa}$ but above the $\mathrm{Tg}$ the rubbery modulus is around $10^{6.8} \mathrm{MPa}$. The Tg of most coil coating top coats is around $25-35^{\circ} \mathrm{C}$. Hence in S.E Asia the topcoats will be above Tg during most of their service life, especially given that moisture from the surrounding humidity could reduce the Tg by $10^{\circ} \mathrm{C}$. Dirt particles vary in size between 1 and $10 \mu \mathrm{m}$ but the ones that stick will be the PM2.5s. Thus dirt retention is dependent upon Tg. Surprisingly dirt pick up is also apparently dependent upon Tg.

Figures 9 and 10 show a correlation between (Tg) and change in colour observed after 1 year exposure in Shenzhen, China. Both washed and unwashed data are presented. There is a clear inverse correlation of the colour change (and hence dirt pick-up) with $\mathrm{Tg}$. The highest $\mathrm{Tg}$ material (a laminate, $\mathrm{Tg}=70^{\circ} \mathrm{C}$ ) retaining the least dirt. Furthermore; the effect of making the surface slightly more hydrophilic by use of an additive, has a dramatic effect on the amount of dirt picked up (unwashed) and retained (washed). 


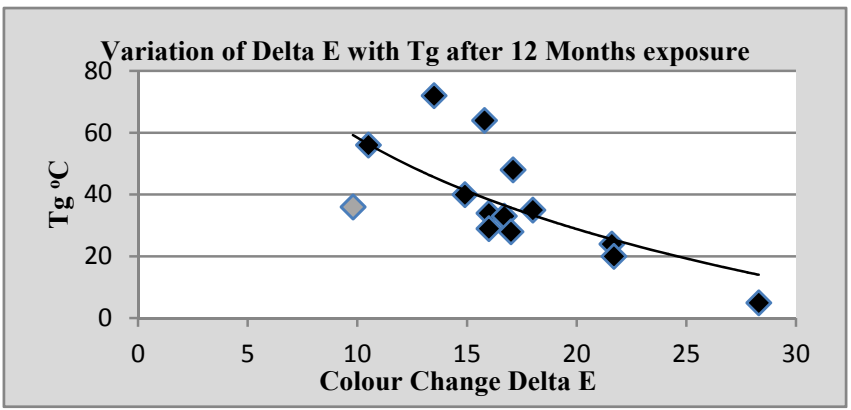

Figure 9: Unwashed colour variation of paints with different Tgs after 12 months exposure in China.

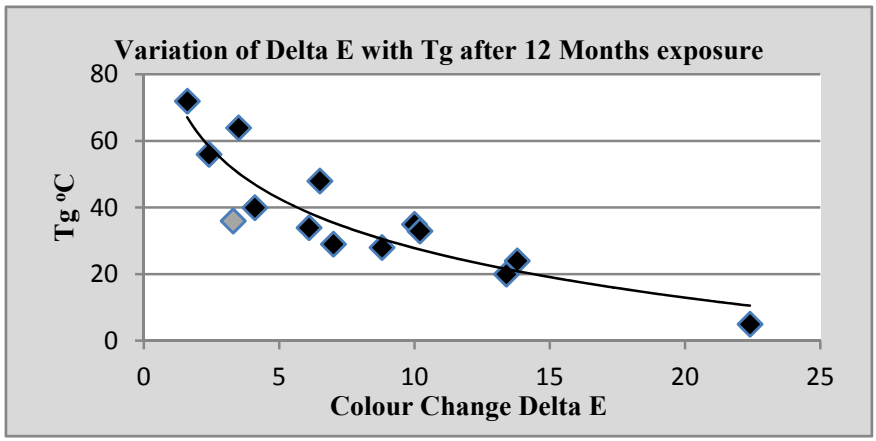

Figure 10: Washed colour variation of paints with different Tgs after 12 months exposure in China.

Figure 11 shows a photograph comparing a standard polyester melamine coating ( Tg between $32-35^{\circ} \mathrm{C}$ ) with a similar system containing an additive and a third highly hydrophilic system. The water contact angles are $80-85^{\circ}, 40-45^{\circ}$ and $5-10^{\circ}$ respectively. This result shows the effectiveness of a hydrophilic surface in resisting dirt deposition.

It is possible to develop two different strategies to increase the polar nature of the surface (hydrophilicity) in order to mitigate dirt pick up. One strategy involves the addition of a small amount of a material that segregates to the surface during cure and renders it hydrophilic after a short exposure to water. The second involves adding a thin third coat of a hydrophilic system. The two approaches are necessary to offer different solutions depending on the coater's application preferences. 


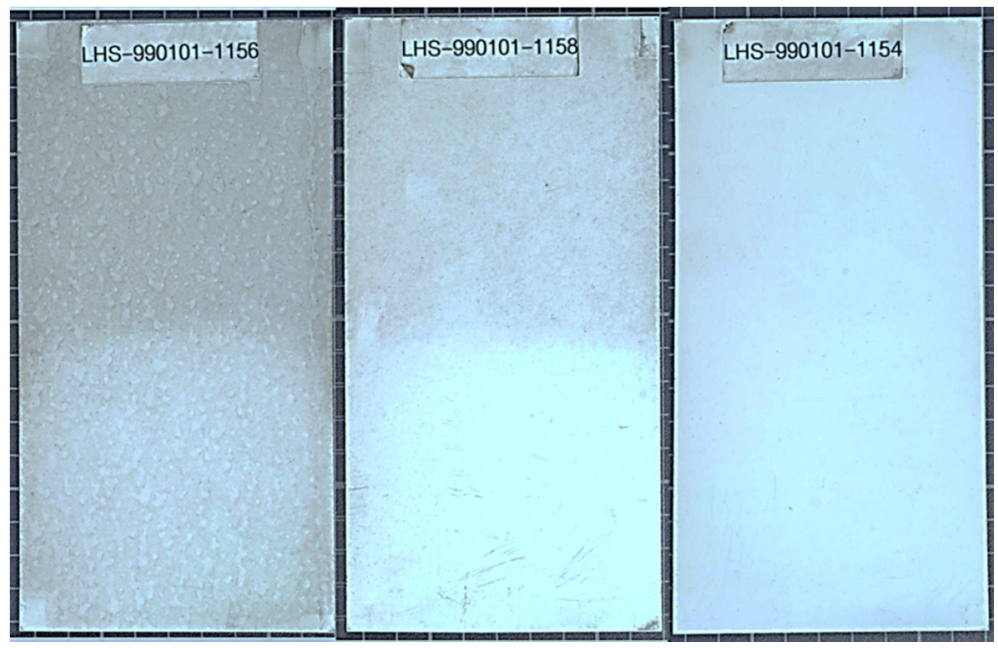

Figure 11: Polyester coatings after 1 year exposure in China LH panel $=$ std, centre panel $=$ with hydrophilic additive, $\mathrm{RH}$ Panel $=$ with hydrophilic third coat.

\section{Conclusion}

More and more resins for coil coatings are being made available (at a development level) that are comprised, at least in part, of bio-sourced raw materials. The properties of the resulting coil coatings are a good match for some of the less demanding applications. Some $100 \%$ bio-sourced resins are available but as yet the durability of the coatings in which they are used is not good enough. However development is moving quickly and there could be products available in a few years' time. The longest delay will be caused by exterior testing which is necessary for warrantees. Use of properly qualified bio sourced coatings should provide the end user with the potential for more credits under schemes such as LEED or BREEAM $^{\circledR}$.

Furthermore, through an in-depth knowledge of materials science, the paint formulator can develop coatings which provide solutions to many of the questions posed by the challenge set by the concept of sustainability. Many of the SMART coatings on offer can be used to claim points in many of the sustainability rating systems that have been put in place by various bodies around the world. One such SMART coating system gives a hydrophilic surface which resists dirt pick up and ensures that we could live in a cleaner environment.

Malaysian Green Buildings Index, awards credits for self-cleaning facades, solar reflecting surfaces and the use of solar thermal technology. Six extra points can be obtained under LEED for Innovation in Design and 2 credits are available under BREEAM: Mat1Materials Specification for choosing systems with low environmental impact. The technology to achieve these credits is available now. 


\section{References}

[1] World Commission on Environment and Development (WCED), Our common future; Oxford (also known as the Brundtland Report): Oxford University Press, p. 43, 1987.

[2] ECCA Sustainable Business Document, 2008.

[3] 2012 Sustainability report of Becker Group.

[4] Smart Coatings Conference, Berlin, 2002.

[5] Dupont Tate and Lyle Bio Products Company Inc., www.duponttate andlyle.com

[6] BioAmber Inc. www.bio-amber.com

[7] Giannakopoulos I. and Taylor A.C., The fracture toughness of polyesterbased coil coatings. Effect of Crosslinker concentration, American Coatings Show, 2012.

[8] www.cagroupltd.co.uk

[9] Barthlott W., Wirtschaft Woche, March 1999.

[10] Ramai D.S. et al. - Colloids and Surfaces A: Physicochem Eng Aspects $165,2000$.

[11] Lowe C., Picking Up Good Vibrations, Rad Tech 1997. 